\title{
Context-Aware and Class Imbalance Invariant Threat Severity Assessment for Heterogeneous loT
}

This paper was downloaded from TechRxiv (https://www.techrxiv.org).

\section{LICENSE}

CC BY-NC-SA 4.0

SUBMISSION DATE / POSTED DATE

$01-11-2021 / 01-11-2021$

\section{CITATION}

A, Nitish; Hanumanthappa, J.; S.P, Shiva Prakash; Krinkin, Kirill (2021): Context-Aware and Class Imbalance Invariant Threat Severity Assessment for Heterogeneous loT. TechRxiv. Preprint. https://doi.org/10.36227/techrxiv.16912504.v1

$\mathrm{DOI}$ 


\section{APPENDIX A}

\section{Root Cause Analysis (RCA) Expert KNOWledgebase}

\section{A. Attack Behaviors, Root Causes, and Fault Scores}

The tables I through IV provide high-level expert knowledge information about the attacks and corresponding fault scores (FS) that is necessary for correlated threat severity assessment from [1]. We extract the information from the resources [4]-[12] mentioned in [2].

TABLE I

RCA Framework for Denial of Service (DoS)

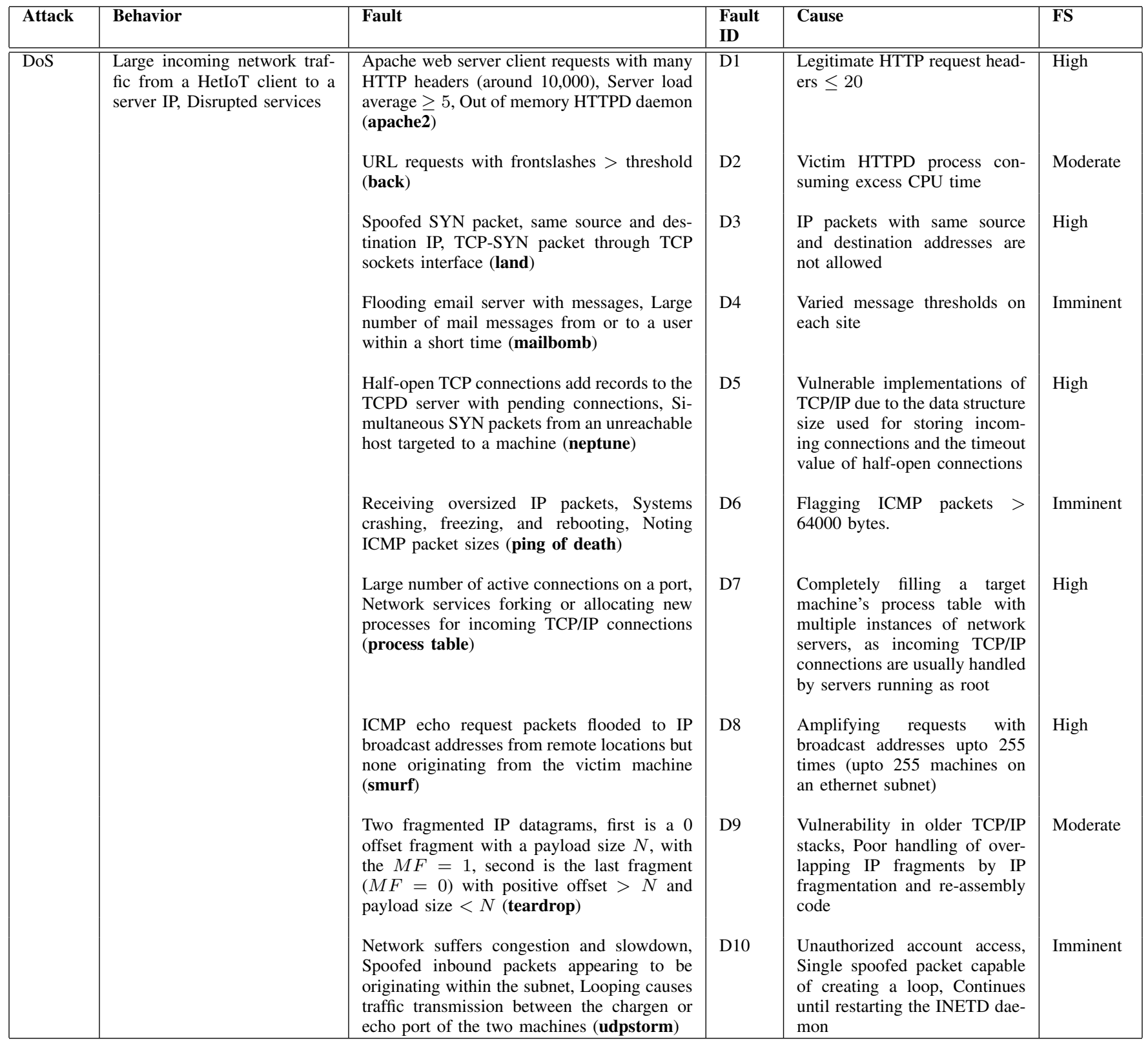


TABLE II

RCA Framework for Distributed Denial of SERvice (DDoS)

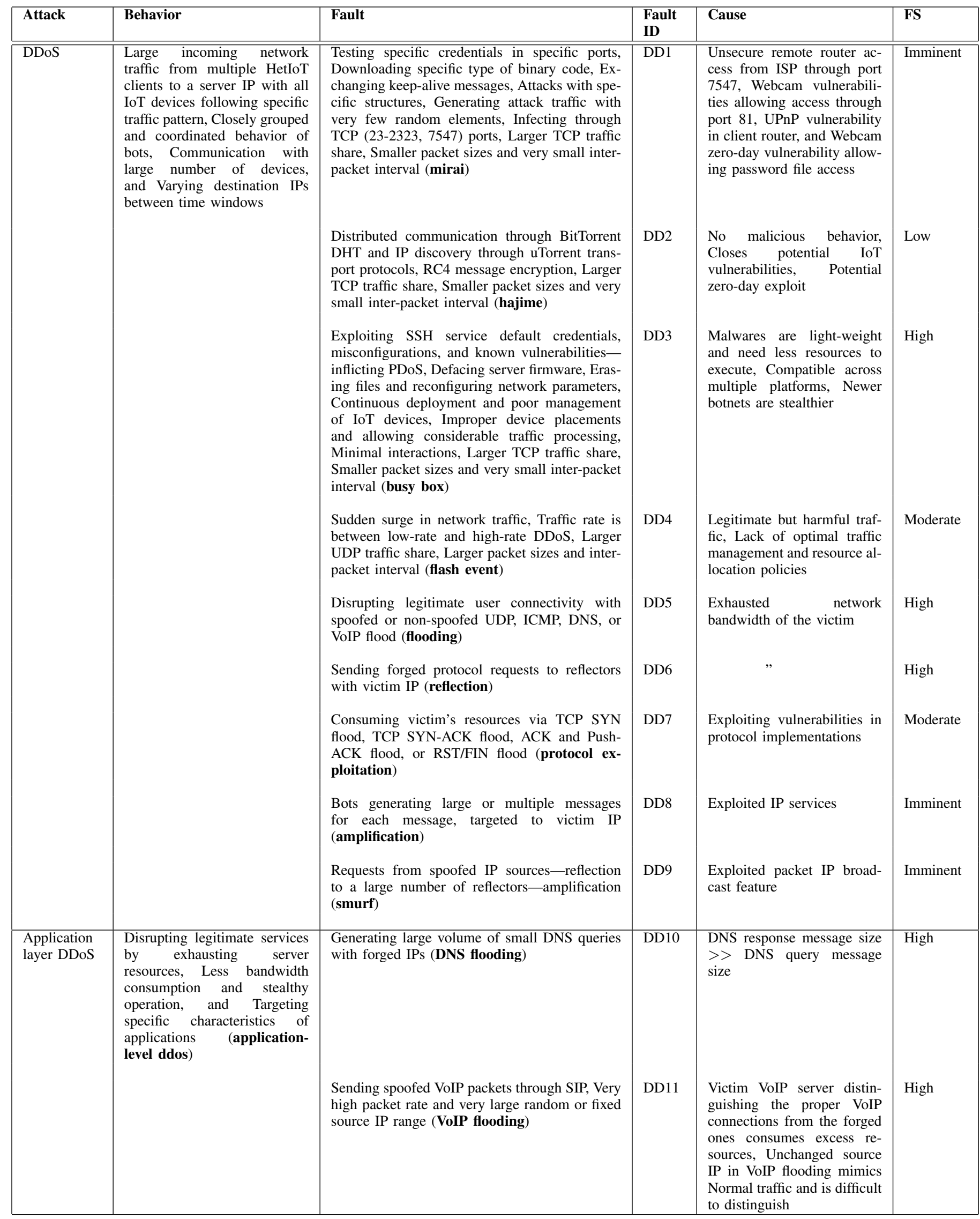




\begin{tabular}{|c|c|c|c|c|c|}
\hline & $\begin{array}{l}\text { Sessions that } \\
\text { high-workload } \\
\text { (asymmetric) }\end{array}$ & $\begin{array}{l}\text { Generating requests to lock up database queries } \\
\text { - like SQL injections (faulty application) } \\
\text { Partial HTTP requests that continuously and } \\
\text { rapidly grow, slowly update, and never close, } \\
\text { continues until all available sockets are taken up } \\
\text { by these requests (slowloris) } \\
\text { HTTP connections held up for a long time } \\
\text { without raising any alarms, similar to slowloris } \\
\text { (fragmentation) } \\
\text { Sending complete HTTP header with "content- } \\
\text { length" field of the post message body for benign } \\
\text { traffic, Sending data to fill the message body at } \\
\text { a rate of one byte every two minutes (slowpost) } \\
\text { Slowly reading the response instead of slowly } \\
\text { sending the requests, Setting a smaller receive } \\
\text { window-size than the target server's send buffer } \\
\text { (slowreading) }\end{array}$ & $\begin{array}{l}\text { DD16 } \\
\text { DD17 }\end{array}$ & $\begin{array}{l}\text { Non-spoofed HTTP get/post } \\
\text { flooding attacks, Hard to de- } \\
\text { tect } \\
\text { Attacker limiting the session } \\
\text { rate of an HTTP attack, By- } \\
\text { pass session rate limitation } \\
\text { defense mechanisms } \\
\text { Maintaining high loads on } \\
\text { the victim server with a } \\
\text { low attack packet rate, At- } \\
\text { tacker nearly invisible to } \\
\text { netflow anomaly detection } \\
\text { techniques, Bypassing deep } \\
\text { packet inspection techniques } \\
\text { by carefully selecting the } \\
\text { HTTP VERB } \\
\text { Poorly designs or improper } \\
\text { integration with databases, } \\
\text { Heavy server resource con- } \\
\text { sumption } \\
\text { Attackers' IP are usually not } \\
\text { spoofed, Limited resources } \\
\text { can affect the server } \\
\text { Non-spoofed bots establish a } \\
\text { valid HTTP connection with } \\
\text { a web server, Fragmenting } \\
\text { legitimate HTTP packets into } \\
\text { tiny fragments and sending } \\
\text { them as slow as the server } \\
\text { time out allows } \\
\text { Server waits for each } \\
\text { message body to be } \\
\text { completed while the attack } \\
\text { grows rapidly } \\
\text { TCP protocol maintains } \\
\text { open connections even in } \\
\text { idle data communication, } \\
\text { Attacker forcing the server } \\
\text { to keep a large number of } \\
\text { connections open }\end{array}$ & Moderate \\
\hline $\begin{array}{l}\text { Botnet-based } \\
\text { DDoS }\end{array}$ & $\begin{array}{l}\text { Controlling the constituent } \\
\text { network devices and making } \\
\text { them behave abnormally }\end{array}$ & $\begin{array}{l}\text { Stealthy C\&C to bot communication via instant } \\
\text { messaging - trinity with UDP, TCP SYN, TCP } \\
\text { ACK, and TCP NUL floods and kaiten with } \\
\text { UDP, TCP, SYN, and PUSH+ACH floods (IRC- } \\
\text { based) } \\
\text { Periodically downloading the instructions using } \\
\text { web requests with encrypted HTTP (port } 80 \text { ) } \\
\text { or HTTPS (port } 443 \text { ) communications without } \\
\text { connecting with C\&C server (web-based) }\end{array}$ & DD21 & $\begin{array}{l}\text { IRC can connect hundreds of } \\
\text { clients via multiple servers, } \\
\text { Using legitimate IRC ports } \\
\text { to send commands to the } \\
\text { bots and can easily hide } \\
\text { his presence because of the } \\
\text { large volume of IRC traffic, } \\
\text { making it difficult to track, } \\
\text { Easy to access the list of all } \\
\text { the available bots instead of } \\
\text { maintaining the list locally } \\
\text { Hide themselves within le- } \\
\text { gitimate HTTP traffic, Con- } \\
\text { figuring and controlling bots } \\
\text { through complex encrypted } \\
\text { PHP scripts and, Difficult to } \\
\text { detect }\end{array}$ & High \\
\hline
\end{tabular}


TABLE III

RCA FRAMEWORK FOR RECONNAISSANCE (RECON)

\begin{tabular}{|c|c|c|c|c|c|}
\hline Attack & Behavior & Fault & $\begin{array}{l}\text { Fault } \\
\text { ID }\end{array}$ & Cause & FS \\
\hline Recon & $\begin{array}{l}\text { Large incoming network traffic } \\
\text { across multiple HetIoT clients } \\
\text { from single or multiple exter- } \\
\text { nal IPs within a specific time } \\
\text { window }\end{array}$ & $\begin{array}{l}\text { ICMP echo requests (ip-sweep) } \\
\text { DNS zone transfer of bruteforce scanning } \\
\text { (mscan) } \\
\text { Traffic via TCP/UDP or via only FIN pack- } \\
\text { ets or via only SYN packets (nmap) } \\
\text { DNS, RPC, unsecure NFS mount points, } \\
\text { fingerd, ruserd, and bootd vulnerabilities on } \\
\text { TCP (70, } 80 \text { FTP, Telnet, SMTP, NNTP, and } \\
\text { UUCP) and UDP (DNS, 177) ports (saint) } \\
\text { Subset of vulnerability checking in the } \\
\text { following order: NFS export, NFS export } \\
\text { via portmapper, NIS password file, REXD, } \\
\text { TFTP file, Remote shell, X server, Write- } \\
\text { able FTP home directory, Sendmail, and } \\
\text { FTP (satan) }\end{array}$ & R4 & $\begin{array}{l}\text { Device replying before a } \\
\text { threshold of requests ex- } \\
\text { poses its IP } \\
\text { Client unable to maintain } \\
\text { active monitoring state for } \\
\text { longer times } \\
\text { Exposed vulnerabilities in } \\
\text { the system }\end{array}$ & Imminent \\
\hline
\end{tabular}

TABLE IV

RCA FRAMEWORK FOR INFORMATION THEFT

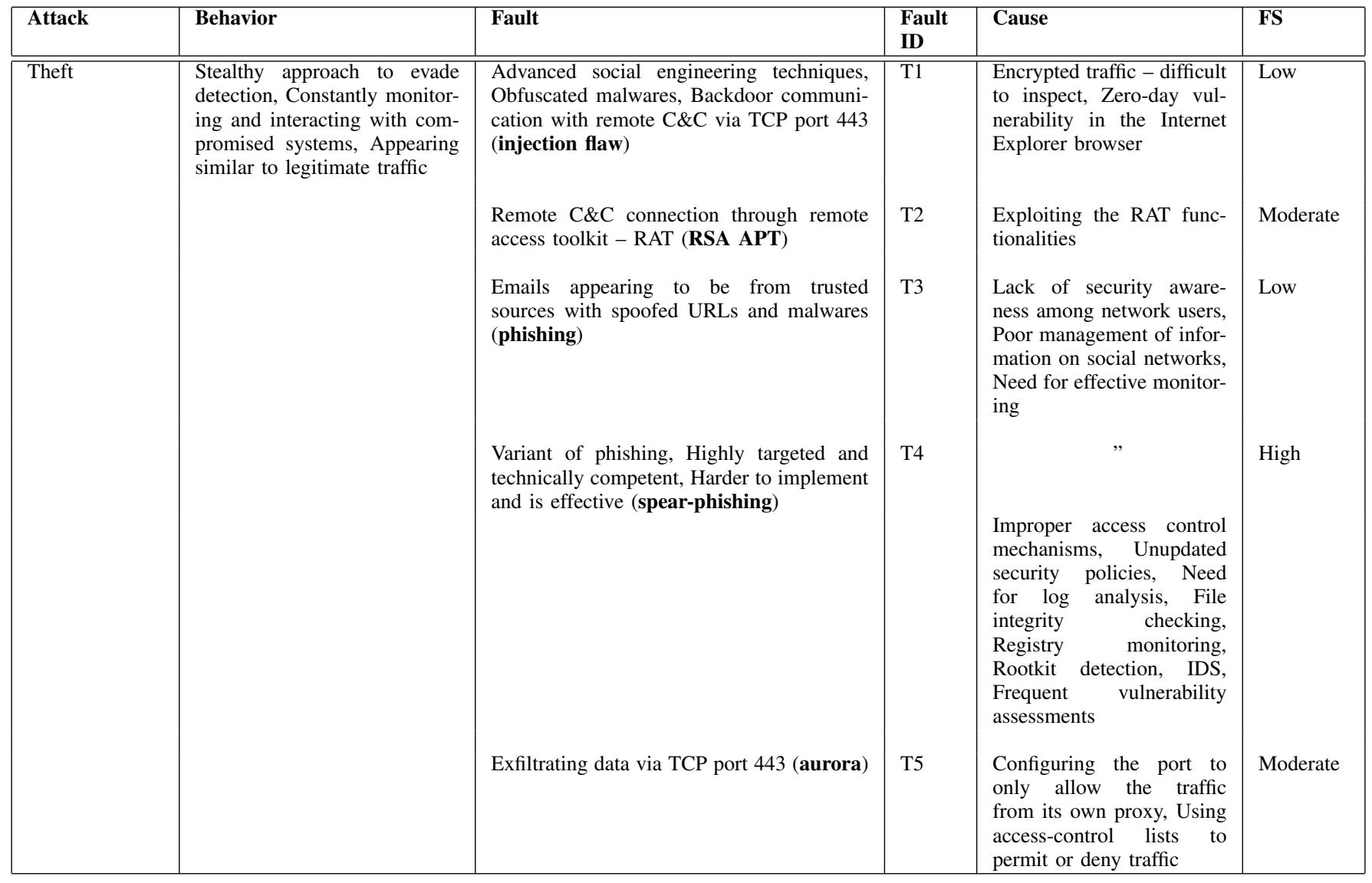




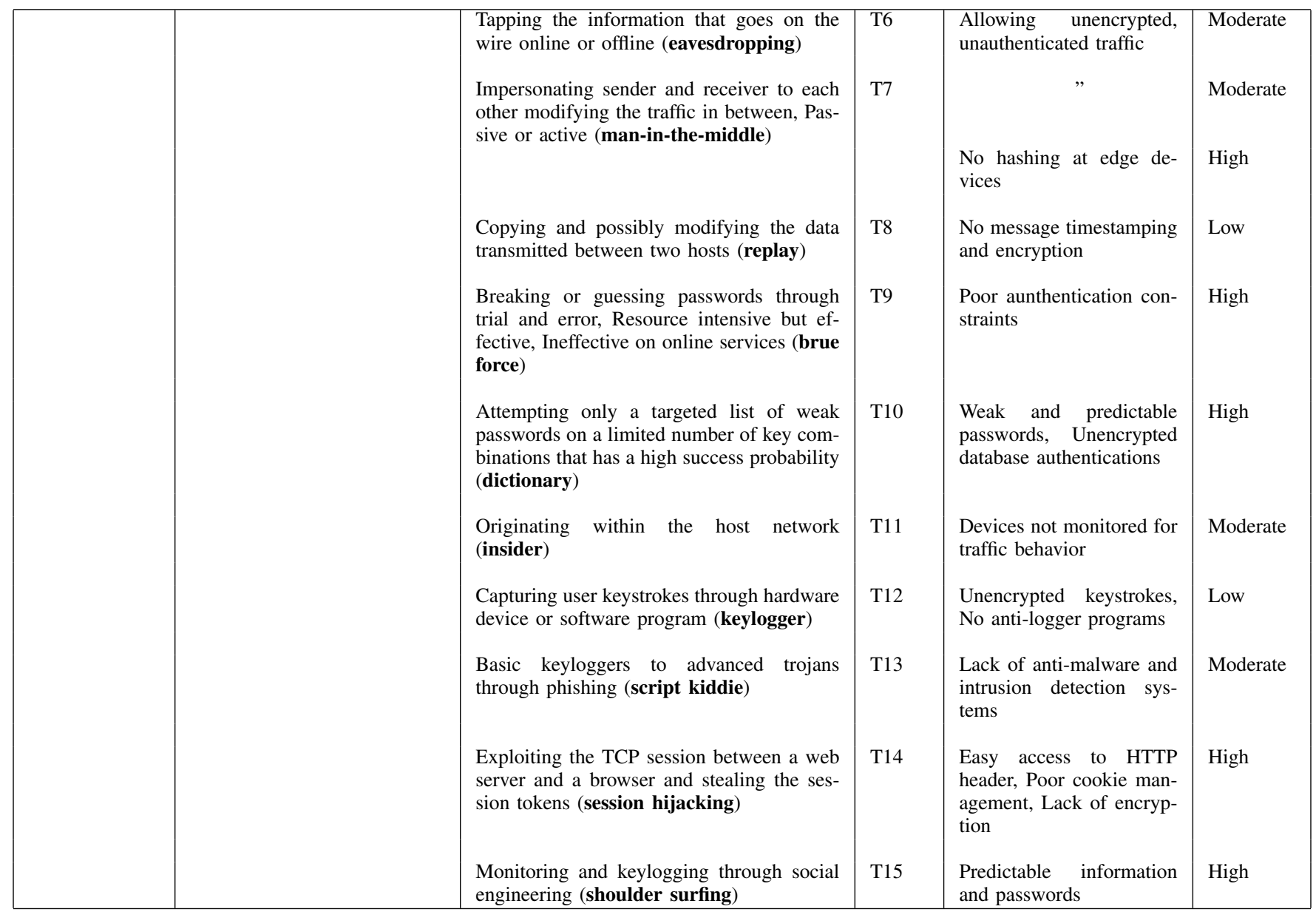




\section{APPENDIX B}

Threat SeVerity ASSESSMEnt (TSA) Expert

KNOWLEDGEBASE

This section presents the proposed $R C A$ and TSA expert knowledge frameworks.

TABLE V

ORdinAl INFERENCE RULES FOR IMMINENT THREAT SEVERITY ASSESSMENT BASED ON TABLE I

\begin{tabular}{|l|l|l|l|}
\hline FS & EoD & RoP & Severity \\
\hline \hline Imminent & Imminent & Imminent & Imminent \\
\hline Imminent & Imminent & High & Imminent \\
\hline Imminent & Imminent & Moderate & High \\
\hline Imminent & Imminent & Low & Moderate \\
\hline Imminent & High & Imminent & Imminent \\
\hline Imminent & High & High & Imminent \\
\hline Imminent & High & Moderate & Moderate \\
\hline Imminent & High & Low & Moderate \\
\hline Imminent & Moderate & Imminent & Imminent \\
\hline Imminent & Moderate & High & High \\
\hline Imminent & Moderate & Moderate & Moderate \\
\hline Imminent & Moderate & Low & Moderate \\
\hline Imminent & Low & Imminent & Imminent \\
\hline Imminent & Low & High & Imminent \\
\hline Imminent & Low & Moderate & High \\
\hline Imminent & Low & Low & Moderate \\
\hline
\end{tabular}

TABLE VI

Ordinal Inference Rules for High Threat SeVerity Assessment BASED ON TABLE I

\begin{tabular}{|l|l|l|l|}
\hline FS & EoD & RoP & Severity \\
\hline \hline High & Imminent & Imminent & Imminent \\
\hline High & Imminent & High & Imminent \\
\hline High & Imminent & Moderate & Moderate \\
\hline High & Imminent & Low & Moderate \\
\hline High & High & Imminent & Imminent \\
\hline High & High & High & High \\
\hline High & High & Moderate & Moderate \\
\hline High & High & Low & Moderate \\
\hline High & Moderate & Imminent & High \\
\hline High & Moderate & High & High \\
\hline High & Moderate & Moderate & Moderate \\
\hline High & Moderate & Low & Moderate \\
\hline High & Low & Imminent & Imminent \\
\hline High & Low & High & High \\
\hline High & Low & Moderate & High \\
\hline High & Low & Low & Moderate \\
\hline
\end{tabular}

TABLE VII

Ordinal InfERENCE RULES FOR Moderate Threat SEVERITy ASSESSMENT BASED ON TABLE I

\begin{tabular}{|l|l|l|l|}
\hline FS & EoD & RoP & Severity \\
\hline \hline Moderate & Imminent & Imminent & High \\
\hline Moderate & Imminent & High & High \\
\hline Moderate & Imminent & Moderate & Moderate \\
\hline Moderate & Imminent & Low & Low \\
\hline Moderate & High & Imminent & High \\
\hline Moderate & High & High & High \\
\hline Moderate & High & Moderate & Moderate \\
\hline Moderate & High & Low & Low \\
\hline Moderate & Moderate & Imminent & High \\
\hline Moderate & Moderate & High & High \\
\hline Moderate & Moderate & Moderate & High \\
\hline Moderate & Moderate & Low & Low \\
\hline Moderate & Low & Imminent & High \\
\hline Moderate & Low & High & High \\
\hline Moderate & Low & Moderate & Moderate \\
\hline Moderate & Low & Low & Moderate \\
\hline
\end{tabular}

TABLE VIII

ORdinAl INFERENCE RULES FOR LOW THREAT SEVERITy ASSESSMENT BASED ON TABLE I

\begin{tabular}{|l|l|l|l|}
\hline FS & EoD & RoP & Severity \\
\hline \hline Low & Imminent & Imminent & Moderate \\
\hline Low & Imminent & High & Moderate \\
\hline Low & Imminent & Moderate & Low \\
\hline Low & Imminent & Low & Low \\
\hline Low & High & Imminent & Moderate \\
\hline Low & High & High & Moderate \\
\hline Low & High & Moderate & Low \\
\hline Low & High & Low & Low \\
\hline Low & Moderate & Imminent & Moderate \\
\hline Low & Moderate & High & Moderate \\
\hline Low & Moderate & Moderate & Low \\
\hline Low & Moderate & Low & Low \\
\hline Low & Low & Imminent & Moderate \\
\hline Low & Low & High & Low \\
\hline Low & Low & Moderate & Low \\
\hline Low & Low & Low & Low \\
\hline
\end{tabular}

\section{APPENDIX C BETA APPROXIMATION}

Theorem C.1 (HetIoT Traffic Behavior Approximation). The Beta probability distribution, based on the Poisson-Gamma joint probability distribution, better approximates the inbound and outbound HetIoT traffic behavior with spatiotemporal dependencies across multiple subnets.

Proof. It is evident that a network traffic dataset consists of both continuous and discrete features, and the Poisson distribution can approximate the discrete network features [3].

If $d_{i}$ where $D=\left\{d_{i}\right\}_{i=1}^{M}, \forall d_{i} \in \mathbb{R}^{N}$ is an inbound or outbound network traffic instance, the accumulation of such events over a unit time interval or volume of connections $(z=1)$ within a subnet is a Poisson process $\mathbb{P}$, formulated as follows [3],[10]:

$$
\begin{aligned}
\mathbb{P}(d ; \lambda z) & =e^{-\lambda z} \frac{(\lambda z)^{d}}{d !} \\
\Rightarrow & \mathbb{P}(d ; \lambda)=e^{-\lambda} \frac{\lambda^{d}}{d !}
\end{aligned}
$$


However, the inherent memorylessness and disjointedness properties of a Poisson process [10] make it insufficient to approximate the spatiotemporal dependencies of the HetIoT traffic that span across multiple subnets over varied time intervals - necessitating a continuous distribution that can capture the said dependencies together-essentially offering new insights into the attack behaviors.

Since the Gamma distribution $\mathbb{G}$ is a conjugate of the Beta distribution, the Poisson-Gamma joint distribution model can solve the above problem by estimating the Poisson parameter $\lambda$ to construct a Markov chain for efficient traffic behavior approximation of the spatiotemporal dependencies taken together; this is formulated as follows [3]:

$$
\mathbb{G}\left(\lambda ; b_{1}, b_{2}\right)=\frac{1}{\Gamma\left(b_{1}\right)} b_{2}^{b_{1}} \lambda^{b_{1}-1} e^{-b_{2} \lambda}
$$

where $b_{1}, b_{2} \in \mathbb{R}^{M}$ are the Gamma distribution parameters and $\Gamma\left(b_{1}\right)=\left(b_{1}-1\right) \Gamma\left(b_{1}-1\right)$ is the corresponding Gamma function.

For the network traffic dataset $D \in \mathbb{R}^{M \times N}$ the Gamma distribution parameters $b_{1}$ and $b_{2}$ are defined as follows:

$$
b_{1}=\frac{[\mathbb{E}(D)]^{2}}{\mathbb{V}(D)} \quad ; \quad b_{2}=\frac{[\mathbb{E}(D)]}{\mathbb{V}(D)}
$$

where $\mathbb{E}$ is the expectation and $\mathbb{V}$ is the variance.

The Beta distribution can approximate the Gamma distribution that occurs naturally at appropriate time intervals between Poisson distributed processes [14].

We complete the proof of the above theorem through the following lemma based on the work presented in [14].

Lemma C.1.1 (Beta Approximation of Poisson-Gamma Joint Distribution). A sample Poisson-Gamma joint probability distributed process with parameters $a$ and $b$ of the form

$$
\mathbb{G}\left(\lambda ; b_{1}, b_{2}\right)=\frac{1}{\Gamma\left(b_{1}\right)} b_{2}^{b_{1}} \lambda^{b_{1}-1} e^{-b_{2} \lambda}
$$

can be approximated from Beta distribution of the form

$$
\mathbb{B}\left(\lambda ; b_{1}, b_{2}\right)=\frac{1}{B\left(b_{1}, b_{2}\right)} \lambda^{b_{1}-1}(1-\lambda)^{b_{2}-1}
$$

resulting in,

$$
\text { Sample }\left(\lambda ; b_{1}, b_{2}\right) \sim \mathbb{B}\left(D ; c_{1}, c_{2}\right)
$$

where $c_{1}$ and $c_{2}$ are the Beta distribution parameters.

Proof. We formulate the Beta distribution approximation of the Poisson-Gamma joint distribution from equations (12) and (13) as,

$$
\mathbb{B}\left(\lambda ; b_{1}, b_{2}\right)=\frac{1}{B\left(b_{1}, b_{2}\right)} \lambda^{b_{1}-1}(1-\lambda)^{b_{2}-1}
$$

where,

$$
B\left(b_{1}, b_{2}\right)=\int_{0}^{1} d^{b_{1}-1}(1-d)^{b_{2}-1} \partial d *=\frac{\Gamma\left(b_{1}\right) \Gamma\left(b_{2}\right)}{\Gamma\left(b_{1}+b_{2}\right)}
$$

*Since the conventional derivative term ' $d$ ' represents a dataset sample, we use the term ' $\partial$ ' in this scenario. is the corresponding Beta function with the parameters $b_{1}$ and $b_{2}$.

Beta distribution can approximate Gamma distribution that occurs naturally at relevant time intervals between Poisson distributed processes.

It follows from [14] that, if $D=\left\{d_{M i}\right\}_{i=1}^{N}$ is a set of Gamma distributed independent random variables having the parameters $b_{1 i}$ and $b_{2}$, then:

$$
\sum_{i=1}^{N}\left(b_{1 i}, b_{2}\right) \Rightarrow b_{1}=\sum_{i=1}^{N} b_{1 i} \quad ; \quad b_{2}=b_{2}
$$

From equation (15), it can be specified (for $N=2$ ) that, we have Gamma parameters $\left(b_{11}, b_{2}\right)$ and $\left(b_{12}, b_{2}\right)$. Then, the ratio $\frac{d_{M 1}}{d_{M 1}+d_{M 2}}$ is a Beta distribution variate with parameters $b_{11}$ and $b_{12}$. i.e.,

$$
\mathrm{P}\left(d_{M 1}, d_{M 2}\right)=\frac{e^{d_{M 1}+d_{M 2}} d_{M 1}^{b_{11}-1} d_{M 1}^{b_{12}-1}}{\Gamma\left(b_{11}\right) \Gamma\left(b_{12}\right)}
$$

Thus, equation (16) has the following distribution [14]:

$$
\begin{aligned}
\mathrm{P}\left(\frac{d_{M 1}}{d_{M 1}+d_{M 2}}\right) & =\int_{0}^{\infty} g(x, y) \partial x \\
& =\frac{1}{B\left(b_{11}, b_{12}\right)} y^{b_{11}-1}(1-y)^{b_{12}-1}
\end{aligned}
$$

where, $B\left(b_{11}, b_{12}\right)$ is a Beta function having Beta distribution with the parameters $b_{11}$ and $b_{12}$.

Substituting the terms $v$ by $\lambda, b_{11}$ by $b_{1}$, and $b_{12}$ by $b_{2}$ in equation (17) results in the following equation:

$$
\begin{aligned}
P\left(\frac{d_{M 1}}{d_{M 1}+d_{M 2}}\right) & =\frac{1}{B\left(b_{1}, b_{2}\right)} \lambda^{b_{1}-1}(1-\lambda)^{b_{2}-1} \\
& =\mathbb{B}\left(\lambda ; b_{1}, b_{2}\right)
\end{aligned}
$$

which is same as equation (14) - the proposed objective.

Therefore, the behavior of the inbound and outbound HetIoT traffic $D$, which follows Poisson-Gamma joint probability distribution, can be well modeled by approximating the corresponding parameters $\lambda, b_{1}$, and $b_{2}$ by the Beta probability distribution; this process has the following formulation:

$$
\text { Sample }\left(\lambda ; b_{1}, b_{2}\right) \sim \mathbb{B}\left(D ; \lambda, b_{11}, b_{12}\right)
$$

where, $\lambda, b_{1}$, and $b_{2}$ are the Poisson-Gamma joint probability distribution parameters, and $b_{11}$ and $b_{12}$ are the Beta distribution parameters.

\section{APPENDIX D}

\section{PERFormance ANAlysis of Algorithms}

Theorem D.1 (Computational Complexity Analysis of CorrELM Algorithm). For a network traffic dataset $D \in \mathbb{R}^{M \times N}$ with $M$ samples and $N$ features and the corresponding expert knowledge $E_{R C A} \in \mathbb{R}^{Q \times 6}$ with $Q$ samples and six features, and $C$ target categories, the computational complexity of the CorrELM algorithm is as follows:

$$
\mathcal{T}_{\text {CorrELM }} \rightarrow \mathcal{O}\left(M\left(L^{2}+Q\right)+L^{3}\right)
$$




$$
\mathcal{S}_{\text {CorrELM }} \rightarrow \mathcal{O}\left(L^{2}+(2 L+Q) C\right)
$$

Proof. We base our implementation of the CorrELM algorithm on the work presented in [13], which shows that the worstcase time and space complexities of the ELM algorithm with $L$ hidden layer neurons and $C$ target categories, respectively, are as follows:

$$
\begin{aligned}
& \mathcal{T}_{E L M} \rightarrow \mathcal{O}\left(M L^{2}+L^{3}\right) \\
& \mathcal{S}_{E L M} \rightarrow \mathcal{O}\left(L^{2}+2 L C\right)
\end{aligned}
$$

The time complexity of correlating expert knowledge $E_{R C A} \in \mathbb{R}^{Q \times 6}$ reduces linearly to

$$
\mathcal{T}_{E_{R C A}} \rightarrow \mathcal{O}(Q)
$$

which follows from expression (i) in the following lemma.

The space complexity of correlating expert knowledge $E_{R C A}$ and the corresponding target category $C$ is

$$
\mathcal{S}_{E_{R C A}} \rightarrow \mathcal{O}(Q C)
$$

Since the CorrELM algorithm classifies $M$ data instances by correlating with $Q$ expert knowledge instances of the matching category $C$, the corresponding time complexity is determined by combining expressions (a) and (c). i.e.,

$$
\mathcal{T}_{\text {CorrELM }} \rightarrow \mathcal{O}\left(M L^{2}+L^{3}+M Q\right)
$$

which follows from lines 12 and 13 in Algorithm 4 and the following lemma.

Similarly, the space complexity of the CorrELM algorithm from expressions (b) and (d) is as follows:

$$
\mathcal{S}_{\text {Corr ELM }} \rightarrow \mathcal{O}\left(L^{2}+2 L C+Q C\right)
$$

Therefore, the computational complexity of the CorrELM algorithm that follows from expressions (e) and (f) is as follows:

$$
\begin{gathered}
\mathcal{T}_{\text {CorrELM }} \rightarrow \mathcal{O}\left(M\left(L^{2}+Q\right)+L^{3}\right) \\
\mathcal{S}_{\text {CorrELM }} \rightarrow \mathcal{O}\left(L^{2}+(2 L+Q) C\right)
\end{gathered}
$$

Corollary D.1.1 (Offline Computational Complexity Analysis of CorrELM Algorithm). During offline training on a sufficiently large dataset, the computational complexity of CorrELM algorithm reduces to that of ELM algorithm. i.e.,

$$
\begin{aligned}
& \mathcal{T}_{\text {CorrELM }}^{o} \approx \mathcal{T}_{E L M} \\
& \mathcal{S}_{\text {CorrELM }}^{o} \approx \mathcal{S}_{E L M}
\end{aligned}
$$

Proof. Let $D=\left\{d_{i}, y_{i}\right\}_{i=1}^{M} \in \mathbb{R}^{M \times N}$ be the network traffic dataset and $E_{R C A}=\left\{k_{j}, y_{j}\right\}_{j=1}^{Q} \in \mathbb{R}^{Q \times 6}$ be the corresponding expert knowledgebase for a matching attack category $C$.
For a sufficiently large network traffic dataset, the corresponding expert knowledgebase is comparatively very smallas a large number of the dataset instances refer to a very small set of expert knowledgebase instances with a matching attack category. Hence, we have

$$
D_{M \times N}>>E_{R C A_{Q \times 6}}
$$

because $\mathbb{R}^{Q \times 6}<<\mathbb{R}^{M \times N}$

Typically, a classifier is trained on an entire (unsampled) large sized network traffic dataset in the background offline. Thus $Q$ has negligible effect on the training time complexity. Therefore, from (j), the expression $(\mathrm{g})$ of the preceding theorem becomes,

$$
\mathcal{T}_{\text {Corr ELM }}^{o} \rightarrow \mathcal{O}\left(M L^{2}+L^{3}\right)
$$

which is same as expression (a), and the expression (h) becomes,

$$
\mathcal{S}_{\text {Corr ELM }}^{o} \rightarrow \mathcal{O}\left(L^{2}+2 L C\right)
$$

which is same as expression (b).

Therefore, from expressions (k) and (l), we can conclude that, for a sufficiently large network traffic dataset during offline training,

$$
\begin{aligned}
& \mathcal{T}_{\text {Corr ELM }}^{o} \approx \mathcal{T}_{E L M} \\
& \mathcal{S}_{\text {Corr ELM }}^{o} \approx \mathcal{S}_{E L M}
\end{aligned}
$$

Theorem D.2 (Computational Complexity Analysis of Threat Severity Algorithm). For $m$ correlated attack alerts and $Q$ corresponding expert knowledge inferences, the computational complexity of the Alert Priority algorithm is,

$$
\begin{aligned}
& \mathcal{T}_{\text {severity }} \rightarrow \mathcal{O}(m Q) \\
& \mathcal{S}_{\text {severity }} \rightarrow \mathcal{O}(Q C)
\end{aligned}
$$

Proof. Algorithm 6 determines the matching fault ID, cause and fault score (FS) for $m C T P$ attack alerts and $Q$ corresponding instances in $E_{R C A}(k) \in \mathbb{R}^{Q \times 6}$, beginning with three instructions (which follows from lines 1-4 in Algorithm 6)

The algorithm continues to find the matching $F S$ in $E_{T S A}(l) \in \mathbb{R}^{27 \times 4}$, resulting in two more instructions each for $27 E_{R C A}$ entries (following from lines 5-7).

Finally, the algorithm returns threat severity for each correlated attack instance.

Thus, the total instruction execution time is as follows:

$$
I_{\text {severity }} \leftarrow m \times Q(2+27 \times 2+1)
$$

Threat severity assessment from $E_{T S A} \in \mathbb{R}^{27 \times 4}$ has a constant space complexity as the expert knowledge table is invariant of input size. Therefore,

$$
\mathcal{S}_{E_{T S A}} \rightarrow \mathcal{O}(1)
$$


Since, the space complexity of the Alert Priority algorithm is the combined space complexities of $E_{R C A}$ and $E_{T S A}$ tables, from expressions (d) and (p), we have:

$$
\mathcal{S}_{\text {severity }} \rightarrow \mathcal{S}_{E_{R C A}}+\mathcal{S}_{E_{T S A}}
$$

Therefore, the resultant computational complexity of alert priority algorithm from expressions (o) and (q) is,

$$
\begin{aligned}
& \mathcal{T}_{\text {severity }} \rightarrow \mathcal{O}(m Q) \\
& \mathcal{S}_{\text {severity }} \rightarrow \mathcal{O}(Q C)
\end{aligned}
$$

\section{APPENDIX E}

\section{ThreAt SEVERITy ASSESSMENT}

The following tables provide attack-wise threat assessments based on the RCA tables. Implementing the ELM classifier algorithm resulted in 46 DoS, 565 DDoS, 182 Recon, and 11 Theft false alerts in total from Table XI of the paper. We present 10 random attack-wise alerts and their assessments in the following tables.

TABLE IX

Threat Severity Assessment of Correlated DoS Alerts

\begin{tabular}{|l|l|l|l|l|l|}
\hline Alert & Fault ID & FS & EoD & RoP & Severity \\
\hline \hline 3 & D2 & Moderate & Low & High & High \\
\hline 7 & D1 & High & Moderate & High & High \\
\hline 16 & D9 & Moderate & Imminent & Moderate & Moderate \\
\hline 12 & D7 & High & Low & Imminent & Imminent \\
\hline 29 & D1 & High & High & High & High \\
\hline 34 & D9 & Moderate & Moderate & Imminent & High \\
\hline 37 & D10 & Imminent & Imminent & Imminent & Imminent \\
\hline 40 & D8 & High & Moderate & Moderate & Moderate \\
\hline 45 & D6 & Imminent & Imminent & Moderate & High \\
\hline 46 & D2 & Moderate & Moderate & High & High \\
\hline
\end{tabular}

TABLE X

Threat SEVErity Assessment of CoRrelated DDoS Alerts

\begin{tabular}{|l|l|l|l|l|l|}
\hline Alert & Fault ID & FS & EoD & RoP & Severity \\
\hline \hline 28 & DD6 & High & Low & High & High \\
\hline 52 & DD12 & High & Low & Low & Moderate \\
\hline 56 & DD19 & Moderate & Moderate & Low & Low \\
\hline 14 & DD7 & Moderate & High & High & High \\
\hline 74 & DD11 & High & High & High & High \\
\hline 88 & DD15 & Moderate & High & Imminent & High \\
\hline 114 & DD17 & Low & Moderate & Low & Low \\
\hline 150 & DD18 & High & Moderate & Low & Moderate \\
\hline 200 & DD20 & High & High & High & High \\
\hline 350 & DD21 & High & Low & Low & Moderate \\
\hline
\end{tabular}

TABLE XI

Threat SeVerity Assessment of Correlated Recon Alerts

\begin{tabular}{|l|l|l|l|l|l|}
\hline Alert & Fault ID & FS & EoD & RoP & Severity \\
\hline \hline 2 & R2 & Moderate & Moderate & Moderate & High \\
\hline 4 & R4 & Imminent & Moderate & Low & Moderate \\
\hline 15 & R1 & Moderate & Moderate & Low & Low \\
\hline 19 & R2 & Moderate & Low & High & High \\
\hline 26 & R3 & High & High & High & High \\
\hline 30 & R5 & High & High & Imminent & Imminent \\
\hline 62 & R4 & Imminent & High & Imminent & Imminent \\
\hline 91 & R1 & Moderate & Moderate & Low & Low \\
\hline 101 & R3 & High & High & High & High \\
\hline 180 & R5 & High & High & High & High \\
\hline
\end{tabular}

TABLE XII

Threat Severity Assessment of Correlated Theft Alerts

\begin{tabular}{|l|l|l|l|l|l|}
\hline Alert & Fault ID & FS & EoD & RoP & Severity \\
\hline \hline 1 & T6 & Moderate & Low & Low & Moderate \\
\hline 2 & T10 & High & Moderate & Low & Moderate \\
\hline 3 & T11 & Moderate & Low & Moderate & Moderate \\
\hline 4 & T9 & High & Moderate & Moderate & Moderate \\
\hline 5 & T3 & Low & Low & Low & Low \\
\hline 6 & T5 & Moderate & Moderate & Low & Low \\
\hline 8 & T14 & High & Moderate & Low & Moderate \\
\hline 9 & T15 & High & Low & Low & Moderate \\
\hline 10 & T8 & Low & High & Low & Low \\
\hline 11 & T2 & Moderate & Low & Low & Moderate \\
\hline
\end{tabular}

\section{REFERENCES}

[1] A. Nitish, J. Hanumanthappa, S. P. S. Prakash, and K. Krinkin, "Expert Knowledge Correlated Threat Evaluation of Intrusion Detection System in Heterogeneous IoT," TechRxiv. Preprint. 2021. [Online]. Available. https://doi.org/10.36227/techrxiv.16722784.v1

[2] N. Koroniotis et al, "Towards the development of realistic botnet dataset in the Internet of Things for network forensic analytics: Bot-IoT dataset," Future Generation Computer Systems, vol. 100, pp. 779-796, 2019.

[3] H. Zhang, X. Yu, and P. Ren, "Deep Adversarial Learning in Intrusion Detection: A Data Augmentation Enhanced Framework,", [Online]. Available. https://arxiv.org/pdf/1901.07949.pdf

[4] R. Doshi, N. Apthorpe, and N. Feamster, "Machine Learning DDoS Detection for Consumer Internet of Things Devices," IEEE Symposium on Security and Privacy Workshops, 2018.

[5] S. Behal and K. Kumar, "Detection DDoS Attacks and Flash Events using Information Theory Metrics - An Empirical Investigation," Computer Communications, 2017.

[6] C. Kolias et al, "DDoS in the IoT: Mirai and Other Botnets," Computer, vol. 50, pp. 80-84, 2017.

[7] N. Hoque et al, "Network Attacks: Taxonomy, Tools and Systems," Journal of Network and Computer Applications, pp. 307-324, 2014.

[8] A. Jesudoss and N.P. Subramaniam, "A Survey on Authentication Attacks and Countermeasures in a Distributed Environment," Indian Journal of Computer Science and Engineering (IJCSE), 2014.

[9] S.T. Zargar et al, "A Survey of Defense Mechanisms Against Distributed Denial of Service (DDoS) Flooding Attacks," IEEE Communications Surveys \& Tutorials, 2013.

[10] M.S. Hoque et al, "An Implementation of Intrusion Detection System using Genetic Algorithm," International Journal of Network Security \& Its Applications (IJNSA), vol. 4, no, 2, 2012.

[11] S. Paliwal and R. Gupta, "Denial-of-Service, Probing \& Remote to User (R2L) Attack Detection using Genetic Algorithm," International Journal of Computer Applications, vol. 60, no. 19, pp. 57-62, 2012.

[12] C. Tankard, "Persistent threats and how to monitor and deter them," Network Security, pp. 16-19, 2011.

[13] A. Akusok, K-M. Bjork, Y. Miche, and A. Lendasse, "High-Performance Extreme Learning Machines: A Complete Toolbox for Big Data Applications," IEEE Access, vol. 3, pp. 1011-1025, 2015.

[14] E.W. Weisstein, "Gamma Distribution," Mathworld, [Online]. Available. https://mathworld.wolfram.com/GammaDistribution.html 